\title{
Bone, Growth Plate and Mineral Metabolism
}

\author{
Outi Mäkitie ${ }^{a}$ and Ola Nilsson ${ }^{b}$ \\ aPediatric Endocrinology and Metabolic Bone Diseases, Children's Hospital, Helsinki University Central Hospital \\ and University of Helsinki, Helsinki, Finland \\ ${ }^{b}$ Center for Molecular Medicine and Pediatric Endocrinology Unit, Department of Women's and Children's \\ Health, Karolinska Institutet and Karolinska University Hospital, Stockholm, Sweden, and Program in \\ Developmental Endocrinology and Genetics, Eunice Kennedy Shriver National Institute of Child Health and \\ Human Development, Bethesda, Md., USA
}

\begin{abstract}
The literature on pediatric bone health and on topics relevant to this chapter is rapidly expanding and changing its focus. Vitamin D, previously regarded important mainly for bone and calcium metabolism, has been suggested to have a role in several other conditions and the vitamin $D$ focus has shifted to cancer, cardiovascular diseases and autoimmunity. This topic has seen fierce debates over the last year. On the other hand, while bone still quite recently was regarded only as a passive partner in mineral metabolism and a target to various calciotropic hormones, it is now evident that the skeleton - apparently the largest endocrine organ in non-obese individuals - produces hormones to regulate mineral availability and actively participates in such fundamental processes as energy metabolism and even fertility. Next-generation sequencing methods are rapidly expanding our knowledge on genetic causes of various rare skeletal conditions and thereby elucidate the complexity of normal skeletal development and function. These findings are anticipated in the future to translate into improved patient care, as already seen in some of the selected papers.
\end{abstract}

\section{Mechanism of the year \\ Skeletons talk - now to the testes}

\section{Endocrine regulation of male fertility by the skeleton}

Oury F, Sumara G, Sumara O, Ferron M, Chang H, Smith CE, Hermo L, Suarez S, Roth BL, Ducy P, Karsenty G Department of Genetics and Development, Columbia University, New York, N.Y., USA.

gk2172@columbia.edu

Cell 2011;144:796-809.

Background: Observations on interactions between bone and the reproductive system have usually focused on the effects of gonadal steroids on bone remodeling. This study explores potential influences by the skeleton on gonadal function.

Methods: Coculture assays, genetically modified cell lines and mouse models were used to study interactions between the skeleton and gonads.

Results: Using coculture assays, the authors demonstrated that osteoblasts are able to induce testosterone production by the testes but do not influence estrogen production by the ovaries. Analyses of cell-specific loss- and gain-of-function models revealed that this endocrine function is mediated by the osteoblast-derived hormone osteocalcin. By binding to a G-protein-coupled receptor in the Leydig cells of the testes, osteocalcin regulates the expression of enzymes required for testosterone biosynthesis and promotes germ cell survival.

Conclusions: This study expands our understanding of the physiological functions of osteocalcin and provides for the first time evidence that the skeleton is a positive endocrine regulator of male fertility.

Based on physiological and clinical observations, it was hypothesized a decade ago that bone mass, energy metabolism, and reproduction might be coordinately regulated. Testing this hypothesis revealed that bone is an endocrine organ favoring whole-body glucose homeostasis and energy expenditure. These functions of bone are mediated by an osteoblast-specific secreted molecule, osteocalcin, that, when uncarboxylated, acts as a hormone favoring $\beta$-cell proliferation, insulin secre- 


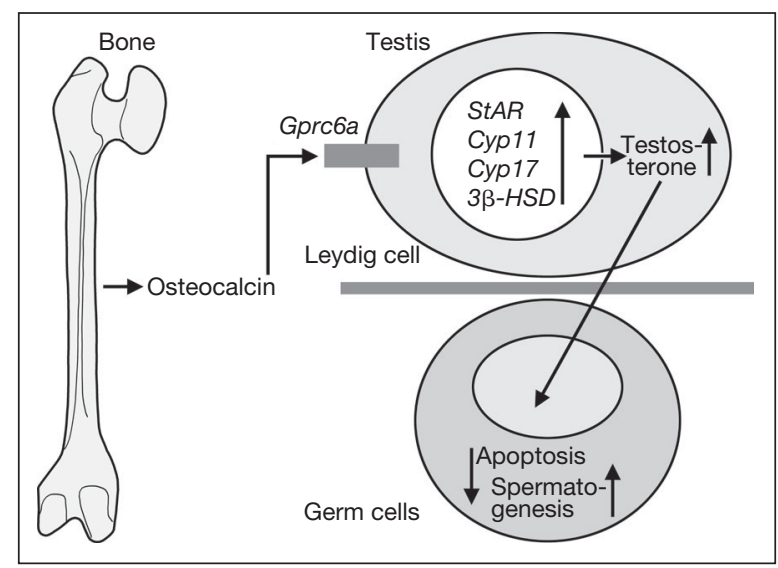

Fig. 1. Osteoblast-derived osteocalcin signals through Gprc6a receptor to promote testosterone production by Leydig cells. Testosterone acts on germ cells and prevents apoptosis and increases spermatogenesis.

tion, and sensitivity and energy expenditure (see the following article) [1, 2]. Now, Oury et al. report of much unexpected observations on the connection between bone mass and reproduction. Recent years have taught us that the bone-forming osteoblasts secrete at least two hormones, FGF23 and osteocalcin, which have a significant function in mineral homeostasis and energy metabolism, respectively. The present study shows that testosterone production by Leydig cells in the testis is directly influenced by the bone-derived hormone osteocalcin. Using cell culture assays the authors demonstrate that a factor secreted by osteoblasts markedly increases testosterone production both by testis explants and by isolated Leydig cells. Such an effect was not seen in osteocalcin-deficient osteoblasts, but could be rescued by the addition of exogenous osteocalcin. Similarly, injection of osteocalcin increased serum testosterone in mice. Osteocalcin-deficient male mice had reduced testis weight, sperm count and circulating levels of testosterone, and had fewer offspring than controls. The researchers subsequently identified a G-protein-coupled receptor, Gprc6a in Leydig cells, through which osteocalcin signals to promote expression of steroidogenic enzymes (fig. 1). Animals lacking osteocalcin had significantly increased serum LH, but this was insufficient to compensate for the silent osteocalcin pathway. The identification of osteocalcin as an important regulator of male fertility and testicular function expands our understanding of the complex mechanisms governing normal androgen biosynthesis in the testis and provides a new window of opportunity for the development of new therapies. Stay tuned, bones may still have more to say...

\section{New paradigms \\ Insulin promotes teamwork in the bone}

\section{Insulin signaling in osteoblasts integrates bone remodeling and energy metabolism}

Ferron M, Wei J, Yoshizawa T, Del Fattore A, DePinho RA, Teti A, Ducy P, Karsenty G

Department of Genetics and Development, College of Physicians and Surgeons, Columbia University, New York, N.Y., USA.

gk2172@columbia.edu

Cell 2010;142:296-308.

Background: The role of insulin receptor in various tissues, especially the bone, is not fully understood. This is also relevant to the osteoblast, a bone-specific cell, since it expresses the insulin receptor and regulates insulin secretion by the secreted protein osteocalcin. 
Methods: The authors used various loss- and gain-of-function models to study insulin signaling in osteoblasts and its effects on osteocalcin and osteoclast function.

Results: Insulin signaling in osteoblasts enhanced osteocalcin activity and influenced glucose homeostasis by promoting the ability of osteoblasts to promote bone resorption. Since bone resorption occurs at a $\mathrm{pH}$ acidic enough to decarboxylate proteins, the authors show that it is the activity of the osteoclast that determines the carboxylation status and function of the osteoblast-derived osteocalcin. Accordingly, increasing or decreasing insulin signaling in osteoblasts promotes or hampers glucose metabolism in a bone resorption-dependent manner in mice and humans.

Conclusions: These data indicate that in a feed-forward loop, insulin signals in osteoblasts activate osteocalcin, which then promotes glucose metabolism. The study identifies insulin signaling in osteoblasts as a key molecular link between bone remodeling and energy metabolism.

Previous studies have demonstrated that the adipose tissue signals via leptin to the skeleton. Lee et al. [1] subsequently showed that bone in turn signals back and regulates energy metabolism [2]. The messenger in this feedback was shown to be osteocalcin, an osteoblast-specific protein, which stimulates insulin expression in pancreatic $\beta$-cells and adiponectin in adipocytes. What then regulates osteocalcin production and how does it communicate with insulin? In the present study the authors provide several lines of evidence to elucidate the detailed steps through which insulin signaling increases production of active (decarboxylated) osteocalcin (fig. 2). They first show that the insulin receptor is a substrate of protein tyrosine phosphatases OST-PTP and PTP1B in mouse and human osteoblasts, respectively. Insulin signaling in osteoblasts decreases expression of osteoprotegerin $(O p g)$, a gene encoding a decoy receptor for RANKL and a negative regulator of osteoclast function. This results in increased Tcirg1 expression and increased osteoclast function. The acidic resorption lacunae provided by active osteoclasts allow osteocalcin decarboxylation. Now the active osteocalcin is ready to exert its metabolic functions in target tissues, including the pancreatic $\beta$-cells, to influence whole-body glucose metabolism. The study sheds some light to the interplay between bone cells and energy metabolism. However, the clinical relevance of these findings remains unknown. Further studies are probably already on the way.

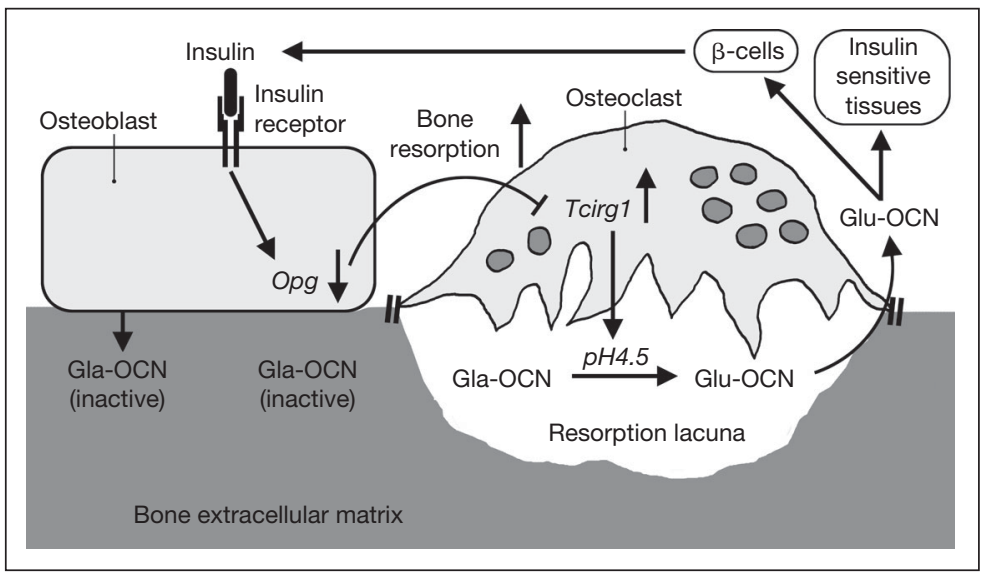

Fig. 2. Insulin signaling in osteoblasts decreases Opg expression. This increases bone resorption, Tcigr1 expression and acidification of the bone extracellular matrix, which promotes osteocalcin decarboxylation. This active osteocalcin influences target tissues to increase insulin secretion and insulin sensitivity. 


\section{Potent inhibition of heterotopic ossification by nuclear retinoic acid receptor- $\gamma$ agonists}

Shimono K, Tung WE, Macolino C, Chi AH, Didizian JH, Mundy C, Chandraratna RA, Mishina Y, Enomoto-Iwamoto M, Pacifici M, Iwamoto M

Department of Orthopedic Surgery, Thomas Jefferson University College of Medicine, The Children's Hospital of Philadelphia, Division of Orthopedic Surgery, Philadelphia, Pa., USA.

Nat Med 2011;17:454-60.

Background: Heterotopic ossification, the formation of ectopic bone within soft tissues, may be induced by surgery or trauma or caused by a rare genetic disorder fibrodysplasia ossificans progressiva (FOP). Ectopic bone formation can have debilitating consequences but no definitive cure has been available. Methods: The authors used mouse embryo mesenchymal cells, mouse models with induced heterotopic ossification and genetically modified mice to explore the efficiency and mechanisms for prevention of heterotopic ossification with nuclear retinoic acid receptor- $\gamma(\mathrm{RAR}-\gamma)$ agonist.

Results: Heterotopic ossification was essentially prevented in mice receiving an RAR- $\gamma$ agonist. Side effects were minimal and no significant rebound effect was seen when treatment was discontinued. To uncover the underlying mechanisms, mouse mesenchymal stem cells were treated with an RAR- $\gamma$ agonist and transplanted into nude mice. This pretreatment prevented formation of ectopic bone masses, suggesting that the cells had lost their skeletogenic potential. The cells became unresponsive to rBMP-2 treatment in vitro. In addition, an RAR- $\gamma$ agonist blocked heterotopic ossification in transgenic mice with a genetic defect comparable to that in patients with FOP.

Conclusions: The findings of this study indicate that RAR- $\gamma$ agonists are potent inhibitors of heterotopic ossification in mice and may thus also be effective against injury-induced and congenital heterotopic ossification in humans.

The process of heterotopic endochondral ossification (HEO) resembles the formation of normal skeleton during embryogenesis but is triggered by inflammatory factors. Local inflammation is followed by the recruitment of skeletal progenitor cells that differentiate into chondrocytes, undergo hypertrophy and are replaced by endochondral bone. Up to $65 \%$ of seriously wounded soldiers develop the disease as well, causing chronic pain, problems with fitting prostheses, deep venous thrombosis, limited motion and other complications [3]. Individuals with the rare genetic condition FOP develop extensive HEO, that is induced by inflammation or trauma, is aggressive and often fatal and is caused by an activating mutation in the BMP type I receptor. Current therapeutic treatments for HEO have targeted various steps of this process but have provided no definitive cure and associated with side effects. The present study was based on the fact that retinoic acid is a potent skeletal teratogen that inhibits chondrogenesis. RAR- $\gamma$ is expressed in chondrogenic cells and chondrocytes and operates in these cells as an unliganded transcriptional repressor; it was therefore chosen as a suitable treatment target for the present study. Shimono et al. provide several lines of evidence showing that a RAR- $\gamma$ agonist does indeed efficiently block HEO, without significant side effects. Importantly, HEO can be prevented after induction, when inflammatory events have already begun. In vitro studies and mouse models showed that both the prechondrogenic and chondrogenic stages of HEO were extremely sensitive to the inhibitory effects of a RAR- $\gamma$ agonist, which blocks BMP signaling and the skeletogenic potential of progenitor cells. These findings provide new hope for individuals suffering from FOP and other conditions with HEO. Further drug development and clinical trials are needed, but the findings suggest that a promising new therapy is underway. 


\title{
Risk of corrected QT interval prolongation after pamidronate infusion in children
}

Rothenbuhler A, Marchand I, Bougneres P, Linglart A

Department of Pediatric Endocrinology and Diabetology, French Reference Center for Rare Diseases of the Calcium and Phosphate Metabolism, Hôpital Saint Vincent de Paul, Assistance Publique/Hôpitaux de Paris, Université Paris Descartes and Institut National de la Santé et de la Recherche Médicale, Unité 986, Paris, France.

J Clin Endocrinol Metab 2010;95:3768-70.

Background: Hypocalcemia, a frequent adverse effect of bisphosphonate infusion, predisposes to cardiac conduction incidents and death. This study investigated effects of pamidronate infusion on ventricular repolarization in children.

Methods: 34 children with cerebral palsy and severe osteoporosis were treated for approximately 1 year with pamidronate (3 times per year). Serum calcium and corrected QT interval (QTc) were measured before and after each pamidronate infusion cycle.

Results: Pamidronate decreased serum calcium in all children, from 2.40 to $2.21 \mathrm{mmol} / \mathrm{l}(\mathrm{p}<0.0001)$ and increased QTc from 390 to $403 \mathrm{~ms}(\mathrm{p}<0.0001)$, with $7.4 \%$ of postinfusion QTc values becoming longer than $440 \mathrm{~ms}$. QTc at baseline was significantly correlated to final QTc $\left(\mathrm{p}<0.0001 ; \mathrm{r}^{2}=0.27\right)$. Conclusion: Because of the observed lengthening in QTc after bisphosphonate infusion, the authors recommend that children treated with pamidronate should be assessed for other possible risk factors of prolonged QT and have a pre- and postinfusion measurement of their QTc.

\section{Administration of the bisphosphonate zoledronic acid during tooth development inhibits tooth eruption and formation and induces dental abnormalities in rats}

\author{
Hiraga T, Ninomiya T, Hosoya A, Nakamura H \\ Department of Histology and Cell Biology, Matsumoto Dental University, Shiojiri, Nagano, Japan. \\ hiraga@po.mdu.ac.jp \\ Calcif Tissue Int 2010;86:502-10.
}

Background: Bisphosphonates (BPs) are potent inhibitors of osteoclastic bone resorption and widely used for the treatment of osteoporosis, also in children with primary and secondary osteoporosis, including osteogenesis imperfecta. The long-term safety of BP use in children has not been established. Clinical and experimental studies have demonstrated that BPs delay or inhibit tooth eruption, resulting in dental abnormalities.

Methods: To determine the effects of BPs on tooth formation, zoledronic acid (ZOL) was injected into 7- and 14-day-old rats, and the development of the mandibular teeth was examined.

Results: X-ray analysis demonstrated that ZOL inhibited the eruption and formation of incisors and molars. Histological examination showed that, in ZOL-treated animals, alveolar bone remained unresorbed around tooth crowns, resulting in injury of ameloblasts and enamel matrix and leading to enamel defects. Furthermore, haphazard proliferation of odontogenic epithelium and mesenchyme associated with primitive tooth structures, which resembles human odontomas, was induced at the basal end of incisors but not around the molars. Tooth ankylosis to alveolar bone was occasionally observed in molars.

Conclusions: The findings of this study suggest that administration of BPs during tooth development may inhibit tooth eruption and formation, resulting in several types of dental abnormalities, possibly attributed to the altered osteoclastic activity.

Bisphosphonates (BPs) are increasingly used in children with osteoporosis. Only a few randomized controlled trials with careful monitoring for possible side effects in children have been published. The two studies referenced here remind us about the potential toxicity and severe side effects of BPs in children. An earlier study in postmenopausal women suggested association between bisphosphonate treatment and atrial fibrillation [4]. Rothenbuhler et al. performed electrocardiograms on 34 
children receiving pamidronate for cerebral palsy-associated secondary osteoporosis. The cumulative pamidronate dose was relatively low, approximately $3.0 \mathrm{mg} / \mathrm{kg}$ over a period of 1 year. All patients received oral calcium and vitamin $D$ supplementation. Despite this, pamidronate infusion resulted in hypocalcemia in $65 \%$. More importantly, pamidronate induced a significant prolongation of the QTC value, the median value increasing from 390 to $403 \mathrm{~ms}$. Consequently, 7\% of postinfusion QTc values were longer than $440 \mathrm{~ms}$, which in children defines prolonged QTc. None of the patients were symptomatic. However, this observation is important and warrants careful monitoring of the patients, as prolonged QTC can result in syncopes, severe arrhythmias, or even sudden death. In the other report, Hiraga et al. have evaluated the impact of BP treatment on tooth development in rats. Zoledronic acid, which is the most potent BP and used also in children, was given in variable doses and dosing regimens to 7- and 14-day-old rats. The results suggest that treatment with BPs during tooth development has the potential to inhibit tooth eruption, impair tooth formation, and induce several types of dental abnormalities. These could be entirely attributed to defective osteoclastic bone resorption. Not only the dose but also timing of treatment (early or late) influenced the dental outcome. Based on these observations, children commencing BP treatment require careful pretreatment evaluation, monitoring during the infusions and follow-up during and after BP therapy to ensure safety. Furthermore, these reports underscore the need for carefully planned controlled trials with BPs in children with primary and secondary osteoporosis.

\section{New treatments}

Anabolic action to combat pediatric secondary osteoporosis?

\section{Intermittent parathyroid hormone administration counteracts the adverse effects of glucocorticoids on osteoblast and osteocyte viability, bone formation, and strength in mice}

Weinstein RS, Jilka RL, Almeida M, Roberson PK, Manolagas SC

University of Arkansas for Medical Sciences, Little Rock, Ark., USA.

weinsteinroberts@uams.edu

Endocrinology 2010;151:2641-9.

Background: Glucocorticoids (GC) act directly on bone cells to decrease osteoblast and osteoclast production, increase osteoblast and osteocyte apoptosis, and prolong osteoclast lifespan.

Methods: The authors used a mouse model and cultured osteoblasts to investigate whether the efficacy of PTH in GC-induced bone disease results from its ability to counteract the direct effects of GCs on bone cells.

Results: GCs were administered to 5- to 6-month-old Swiss-Webster mice for 28 days. The treatment increased osteoblast and osteocyte apoptosis and decreased osteoblast number, activation frequency, and bone formation rate, resulting in decreased bone mineral density and bone strength. Conversely, daily PTH injections decreased osteoblast and osteocyte apoptosis and increased osteoblast number, activation frequency, bone formation rate, bone mineral density, and bone strength. When PTH was given with GCs, all the adverse effects of GC excess on bone were prevented. Similarly, in cultured osteoblasts, PTH attenuated GC-induced adverse effects on osteoblast survival and Wnt signaling via an Akt phosphorylation-dependent mechanism.

Conclusions: The authors conclude that intermittent PTH administration directly counteracts the key pathogenetic mechanisms of GC excess on bone, providing an explanation for the efficacy of PTH treatment against GC-induced osteoporosis.

GC-associated skeletal pathology is characterized by increased osteoblast and osteocyte apoptosis, decreased osteoblast number and bone formation rate, but normal osteoclast number. These features suggest that GC-induced osteoporosis should be treated with anabolic agents rather than with bisphosphonates, which mainly target the osteoclast population. In this study, intermittent PTH administration was used in GC-treated mice. The authors show that daily injections of PTH directly target the key pathogenetic mechanisms associated with GC osteoporosis. Intermittent PTH also 
increased the lifespan of mature osteoblasts by decreasing the prevalence of osteoblast apoptosis; this antiapoptotic effect of PTH was responsible for the increase in wall width, trabecular width, bone formation rate, and bone mass. Continuous PTH excess, e.g. in hyperparathyroidism, increases bone resorption and bone loss. In contrast, intermittently (usually as daily injections) given PTH increases bone formation. Recombinant human PTH 1-34 is used for the treatment of severe osteoporosis in adults. GC-induced osteoporosis is a prevalent problem even in the pediatric patient population with chronic illness. However, due to occurrence of osteosarcomas and death in early rat toxicology studies [5], PTH 1-34 has been contraindicated in children. These toxicology studies were performed at supraphysiological dosage throughout the lifetime of rats and therefore extrapolation of the findings to potential pediatric patients is problematic. Since PTH 1-34 has already been successfully used in children with hypoparathyroidism, maybe it could in the future be considered also for the treatment of severe GC-induced osteoporosis in selected pediatric patient groups. The present arsenal to combat pediatric GC-induced osteoporosis is severely limited.

\title{
New genes \\ Success with next-generation sequencing in early-onset osteoporosis
}

\section{Mutations in NOTCH2 cause Hajdu-Cheney syndrome, a disorder of severe and progressive bone loss}

\author{
Simpson MA, Irving MD, Asilmaz E, Gray MJ, Dafou D, Elmslie FV, Mansour S, Holder SE, Brain CE, Burton BK, Kim \\ KH, Pauli RM, Aftimos S, Stewart H, Kim CA, Holder-Espinasse M, Robertson SP, Drake WM, Trembath RC \\ Division of Genetics and Molecular Medicine, King's College London School of Medicine, Guy's Hospital, London, \\ UK.
}

Nat Genet 2011;43:303-5.

Background: Hajdu-Cheney syndrome is an autosomal dominant multisystem disorder characterized by severe bone loss. The underlying molecular pathology has remained unknown.

Methods: The authors used an exome-sequencing strategy to identify the disease causing genetic defect in Hajdu-Cheney syndrome.

Results: Whole-exome sequencing of 3 unrelated affected individuals revealed NOTCH2 variants in all subjects. Subsequently, NOTCH2 sequencing indentified heterozygous mutations in 11 other patients with Hajdu-Cheney syndrome. All the mutations were predicted to lead to the premature truncation of NOTCH2 with either disruption or loss of the C-terminal proline-glutamate-serine-threonine-rich proteolytic recognition sequence, the absence of which has previously been shown to increase NOTCH signaling.

Conclusions: These findings suggest that Hajdu-Cheney syndrome is caused by increased NOTCH signaling activity.

The Notch pathway has been implicated in multiple aspects of skeletal development and bone homeostasis. This highly conserved signaling system has an important role in organogenesis and tissue renewal. Its intact function is needed for cell-cell communication and can specify diverse cellular events, including proliferation, differentiation, apoptosis and stem cell maintenance. Notch signaling is required for osteoblast differentiation and osteoclast activity [6]. Four different transmembrane Notch receptors (NOTCH1-4) have been characterized; the ligands are also mostly transmembrane proteins and the signaling cascade is activated by direct cell-to-cell contact. Mutations in Notch signaling components have previously been associated with human skeletal dysplasias such as spondylocostal dysostosis and Alagille syndrome. In this paper, Simpson et al. show that aberrant Notch signaling is the cause of Hajdu-Cheney syndrome, a rare disorder with progressive bone loss and acro-osteolysis, craniofacial anomalies and renal cysts. With only 3 unrelated affected individuals and the whole-exome sequencing strategy the researchers were able to show that NOTCH2 mutations underlie Hajdu-Cheney syndrome. The finding was confirmed by identification of similar mutations in 11 other affected individuals. Furthermore, Isidor et al. report in the same issue of Nature Genetics [7] NOTCH2 mutations in another cohort of 5 patients with Hajdu-Cheney syndrome; they too used 
the exome-sequencing approach. All NOTCH2 mutations were clustered in the final exon of the gene, interrupted the coding sequence and were predicted to result in increased Notch signal transduction. This study confirms the significance of intact Notch signaling in skeletal homeostasis. Furthermore, it shows the amazing power of exome-wide sequencing strategy in the identification of causative gene defects in rare inherited conditions. Large-scale genome-wide association studies, carried out in search for the genetic causes of osteoporosis, have identified several gene variants, each with only a minor effect on bone health $[8,9]$. The study by Simpson et al. takes another approach: by identifying the genetic pathology behind a rare inherited condition with severe early-onset osteoporosis, one may discover genes that are likely to be relevant to normal bone health also in the general population.

\section{Exome sequencing identifies truncating mutations in human SERPINF1 in autosomal-recessive osteogenesis imperfecta}

Becker J, Semler O, Gilissen C, Li Y, Bolz HJ, Giunta C, Bergmann C, Rohrbach M, Koerber F, Zimmermann K, de

Vries P, Wirth B, Schoenau E, Wollnik B, Veltman JA, Hoischen A, Netzer C

Institute of Human Genetics, University of Cologne, Cologne, Germany.

Am J Hum Genet 2011;88:362-71.

Background: Osteogenesis imperfecta (OI) is a heterogeneous genetic disorder characterized by bone fragility and low bone mass. OI is usually inherited as an autosomal dominant condition and caused by mutations in the genes encoding type I collagen. Mutations in six other genes have recently been associated with autosomal recessive forms of OI.

Methods: The authors excluded mutations in all known OI genes in a patient with severe OI and consanguineous parents. They then sequenced the exome (approx. 18,600 genes) using the next-generation sequencing methods. A total of 26,922 variations from the human reference genome sequence were subjected to several filtering steps. In addition, homozygous regions were identified using the obtained SNP data.

Results: A single homozygous truncating mutation, affecting SERPINF1 on chromosome 17p13.3, within a homozygous stretch of $2.99 \mathrm{Mb}$ remained as a likely disease-causing mutation. The affected brother of the index patient also was homozygous for the mutation. Subsequent studies showed two different homozygous truncating SERPINF1 mutations in two other unrelated patients with severe OI and parental consanguinity. Collagen analyses with cultured dermal fibroblasts showed no evidence for impaired collagen folding, posttranslational modification, or secretion. SERPINF1 encodes pigment epithelium-derived factor (PEDF), a secreted glycoprotein of the serpin superfamily. PEDF is one of the strongest inhibitors of angiogenesis in humans and may have a role in neovascularization in regions of active bone formation.

Conclusions: This study identifies a new gene involved in the pathogenesis of OI. The specific role of PEDF in human bone homeostasis remains to be elucidated in future studies.

The genetic background of $\mathrm{OI}$ is growing much more complex than previously thought. Some steps in this evolving story have been reviewed in previous Yearbooks [10,11]. Most of the patients with OI have disease-causing heterozygous mutations in COL1A1 and COL1A2, encoding the two $\alpha$ chains of the major bone matrix protein, type I collagen. Six other genes have recently been implicated in autosomal recessive forms of OI. Three of these genes (CRTAP, LEPRE1, PPIB) encode components of the prolyl 3-hydroxylation complex, which is located in the rough endoplasmatic reticulum and has a role in type I collagen $\alpha 1$ chain modification. OSX encodes a transcription factor which is assumed to regulate osteoblast differentiation. SERPINH1 and FKBP10 encode collagen chaperones with a potential role in monitoring the final integrity of the type I collagen triple helix. In this paper the authors describe three mutations in SERPINF1 that are predicted to result in null alleles due to nonsensemediated mRNA decay or impaired secretion of the mutated protein. PEDF, the protein encoded by SERPINF1, is thought to counteract the effect of vascular endothelial growth factor, VEGF, which stimulates angiogenesis and neovascularization in the bones. Based on functional observations in the present study and those reported previously it seems that the severe OI phenotype in patients with SERPINF1 mutations results from mechanisms that are primarily independent of alterations in type I collagen synthesis or intracellular processing. Rather, the pathogenesis is likely to involve neovascularization, since the balance between PEDF and VEGF is thought to be important at sites of active 
bone formation. This report highlights the complexity of the processes behind normal bone formation and maintenance of skeletal integrity - pathology goes far beyond type I collagen.

\author{
New mechanism \\ Rapid growth plate fusion induced by loss of PTH/PTHrP receptor
}

\title{
Parathyroid hormone/parathyroid hormone-related protein receptor signaling is required for maintenance of the growth plate in postnatal life
}

\author{
Hirai T, Chagin AS, Kobayashi T, Mackem S, Kronenberg HM \\ Endocrine Unit, Massachusetts General Hospital and Harvard Medical School, Boston, Mass., USA. \\ hkronenberg@partners.org \\ Proc Natl Acad Sci U S A 2011;108:191-6.
}

Background: In fetal growth cartilage, Indian hedgehog (Ihh), secreted by hypertrophic chondrocytes, stimulates parathyroid hormone (PTH)-related protein $(\mathrm{PTHrP})$ and signals through the PTH/PTHrP receptor (PPR) to inhibit differentiation of proliferative to hypertrophic chondrocytes, thereby completing a feedback loop that controls the number of cells in the proliferative pool. The importance of this mechanism during postnatal growth is less well established.

Methods: Tamoxifen-inducible and cartilage-specific knockout of PPR was generated by the crossing of tamoxifen-inducible collagen type 2 - Cre and floxed PPR mouse lines. Using this model, PPR was targeted specifically in chondrocytes by a single tamoxifen injection at postnatal day 3 .

Results: Inactivation of the PPR in chondrocytes of 3-day-old mice resulted in a dramatic decline in proliferation and accelerated hypertrophic differentiation of chondrocytes, followed by vascular invasion and growth plate fusion within 7 days. Inhibition of hypertrophic cell apoptosis by a low-phosphate diet prevented the disappearance of the growth plate, but not the decline in chondrocyte proliferation or the accelerated hypertrophic differentiation.

Conclusions: The PTH/PTHrP receptor is crucial for maintenance of the growth plate during postnatal growth.

The importance of the Indian hedgehog (Ihh)-parathyroid hormone (PTHrP) feedback loop for bone development, first indicated by human syndromes and later carefully dissected using transgene and gene targeting strategies, is well established for fetal, but not postnatal, bone development. PTHrP is produced by periarticular chondrocytes and adjacent perichondrial cells in fetal life, whereas the PTH/PTHrP receptor (PPR) is produced by proliferating chondrocytes. PTHrP regulates the length of the columnar region by allowing continued proliferation of columnar chondrocytes and suppressing their terminal differentiation into postmitotic hypertrophic chondrocytes in late fetal growth plates [12]. In this article the authors induce cartilage-specific targeting of PPR in the postnatal growth plate in order to test the hypothesis that the Ihh-PTHrP feedback loop is important for postnatal growth plate and the results are striking. When PPR is knocked out in juvenile mice that do not normally fuse their growth plates until the very end of their lifespan, all growth plates fuse within 7 days. These findings do establish the importance of the Ihh-PTHrP feedback loop during postnatal growth. The growth plate disappearance described here may also be relevant to certain forms of human growth plate closure. Early growth plate closure, contributing to brachydactyly and short stature, occurs in children with AHO caused by haploinsufficiency of PTH receptor-related Gs $\alpha$. Because PTHrP activates the PPR and then Gs $\alpha$, and because the KO of Gs $\alpha$ in the growth plate of mice leads to accelerated chondrocyte differentiation resembling that of the PPR KO [13], it is plausible that the growth plate closure in AHO and human heterozygosity for the PTHrP gene result from processes like those demonstrated here. 


\section{Insulin resistance secondary to a high-fat diet stimulates longitudinal bone growth and growth plate chondrogenesis in mice}

Wu S, Aguilar AL, Ostrow V, De Luca F.

Section of Endocrinology and Diabetes, St Christopher's Hospital for Children, Department of Pediatrics, Drexel University College of Medicine, Philadelphia, Pa., USA.

francesco.deluca@drexelmed.edu.

Endocrinology 2011;152:468-75.

Background: Overweight children are often more insulin-resistant and taller than normal-weight peers, suggesting that insulin may contribute to obesity-associated growth acceleration.

Methods: In order to determine whether insulin resistance and secondary hyperinsulinemia contributes to growth acceleration of obesity, juvenile mice were provided standard chow or a high-fat diet and administrated pioglitazone or vehicle.

Results: After 6 weeks' treatment, mice feed a high-fat diet had greater body and tibial growth, tibial growth plate height, and serum insulin levels than those of standard chow-fed mice. Pioglitazone did not affect the weight gain induced by a high-fat diet, but did normalize the insulin levels and abolished the increased longitudinal bone growth detected in mice fed a high-fat diet and administered vehicle. In addition, insulin increased growth of cultured metatarsal bones and cultured chondrocyte proliferation, differentiation, an effect that was abolished by small interfering RNA targeting the insulin receptor.

Conclusions: Insulin resistance, not obesity, is associated with the increased skeletal growth of insulinresistant obese mice fed a high fat diet. This observation in combination with the finding that insulin signaling through the insulin receptor stimulates chondrocyte proliferation suggests that increased insulin signaling makes a significant contribution to the accelerated skeletal growth commonly observed in overweight children.

Overweight children grow faster, have accelerated bone maturation and start puberty earlier compared to normal weight peers. Because overweight children are often insulin-resistant, it has also been hypothesized that their elevated serum insulin levels are mechanistically linked to skeletal growth. In this article, mice fed a high-fat diet develop overweight, insulin resistance, hyperinsulinemia and accelerated longitudinal bone growth. In overweight mice receiving an insulin sensitizer, insulin levels as well as linear bone growth normalized. This finding in combination with in vitro studies suggests that insulin directly promotes growth plate chondrogenesis and that hyperinsulinemia is a major mediator of obesity-associated growth acceleration. The decreased longitudinal bone growth in mice treated with pioglitazone may thus be an unwanted effect of insulin sensitization since it may uncouple linear growth from weight gain and make the relative overweight even worse. Although animal studies cannot fully elucidate the complex network of genetic, hormonal, and environmental mechanisms governing human growth, these findings suggest a causative role for insulin resistance and a direct effect of insulin through its receptor in the obesity-related accelerated statural growth and skeletal maturation. 


\title{
The role of estrogen receptor- $\alpha$ in growth plate cartilage for longitudinal bone growth
}

\author{
Borjesson AE, Lagerquist MK, Liu C, Shao R, Windahl SH, Karlsson C, Sjogren K, Moverare-Skrtic S, Antal MC, Krust \\ A, Mohan S, Chambon P, Savendahl L, Ohlsson C \\ Centre for Bone and Arthritis Research, Institute of Medicine, University of Gothenburg, Sahlgrenska Academy, \\ Goteborg, Sweden. \\ claes.ohlsson@medic.gu.se \\ J Bone Miner Res 2010;25:2690-700.
}

Background: In humans, estrogen signaling through estrogen receptor $\alpha(\mathrm{ER} \alpha)$ enhances the pubertal growth spurt and is also required for normal cessation of growth and growth plate fusion. Consequently, untreated patients that lack estrogen production or one patient with a lack of signaling through ER $\alpha$ show diminished pubertal growth spurt, lack of growth plate fusion and continued longitudinal bone growth well into the third decade of life, resulting in tall stature. This study evaluated whether these effects are mediated through a direct effect of estrogen signaling in growth plate chondrocytes or through systemic effects.

Methods: The authors targeted ER $\alpha$ globally or specifically in cartilage and studied effects on longitudinal bone growth, growth plate function and structure.

Results: As previously reported, global ER $\alpha$ inactivation results in decreased growth until 4 months of age, associated with alterations in the GH/IGF-I axis, an effect not seen in mice lacking ER $\alpha$ in cartilage only. After 4 months, growth is limited in wild-type mice whereas cartilage-specific ER $\alpha$ knockout mice continue to grow resulting in an increased body and femur length at 12 months. In addition, high-dose estradiol treatment of adult mice reduced chondrocyte proliferation and growth plate height in wildtype, but not in cartilage-specific $\mathrm{ER} \alpha$ knockout mice. Whether global $\mathrm{ER} \alpha$ knockout mice continue to grow after 4 months is not reported.

Conclusions: Mice lacking $\mathrm{ER} \alpha$ in chondrocytes grow normally during early life and sexual maturation, but continue to grow well beyond the age when growth normally stops, suggesting that ER $\alpha$ signaling in growth plate chondrocytes is important for growth cessation.

In humans, low estrogen levels enhance skeletal growth during the pubertal growth spurt, whereas high $E_{2}$ levels during late puberty suppress growth. The effect of sex steroids on human growth is mostly understood from patients with rare mutations. Gene targeting of estrogen receptors in mice have often produced conflicting and confusing results. Nevertheless, some lessons will not be possible to address in humans and thus have to be addressed in animal models. The authors produced mice lacking ER $\alpha$ expression in cartilage and find that these animals have normal linear growth during the first 4 months of life - the equivalent of childhood and puberty in humans. After 4 months of age, growth of normal mice is limited, whereas mice lacking ER $\alpha$ in cartilage continue to grow. This finding does not only demonstrate that estrogen signaling through ER $\alpha$ in growth plate chondrocytes is important for normal growth cessation in mice, but the finding is also similar to the lack of growth cessation in the only man reported with a truncated ER $\alpha$ receptor, thus suggesting that not only the effect but also the mechanism has been conserved during the 90 million years of evolution separating primates and rodents. 


\section{The micro-RNA-processing enzyme Dicer is dispensable for somite segmentation but essential for limb bud positioning}

Zhang Z, O’Rourke JR, McManus MT, Lewandoski M, Harfe BD, Sun X

Laboratory of Genetics, University of Wisconsin-Madison, Madison, Wisc., USA.

xsun@wisc.edu

Dev Biol 2011;351:254-65.

Background: Small non-coding micro-RNAs (miRNAs) encoded in the genome regulate gene expression at the posttranscriptional level and were discovered for their role in the control of developmental timing. First transcribed as long RNA transcript, miRNA has to be processed in several steps into mature miRNA in order to be capable of inducing mRNA degradation or translational repression of mRNAs. Dicer is an enzyme that is required for the generation of mature miRNAs.

Aim and Methods: To determine the role of miRNAs in mesoderm development including somite formation and limb bud development. Dicer was specifically targeted in the mesoderm cell lineage by crossing of $\mathrm{T}$ (Brachyury)-Cre and floxed dicer mouse strains.

Results: Mutant mice exhibited a reduced anterior-posterior axis and death 2-3 days after dicer was inactivated. At death, somite numbers were normal, demonstrating that the molecular machineries required for establishing segmentation, including clock and wave front, are not affected. However, somite size was found to be reduced and later-formed somites were caudalized. Outside of the paraxial mesoderm, the timing and position of hindlimb bud initiation delayed and shifted. Consistent with this finding, gene expression changes critical for limb positioning, including changes in Hand2, Tbx3, and Gli3, was shifted and delayed accordingly.

Conclusions: In mouse embryonic mesoderm, miRNAs are required for proper limb bud positioning.

Regulation of gene expression is an important mechanism to control a variety of cellular functions, not the least during development. However, regulation of transcription is often not precise enough and appears to need fine tuning. This is accomplished by miRNAs and fine tuning of gene expression appears to be especially important during embryonic morphogenesis and differentiation. Here, the authors knockout dicer, and thereby eliminate the endogenous production of mature miRNAs, in the mesoderm cell lineage and demonstrate that miRNA is not essential for the initiation of the limb bud, but well for its timing and positioning.

\section{Concepts revised \\ Time to reclassify pseudohypoparathyroidism?}

\section{Functional characterization of GNAS mutations found in patients with pseudohypoparathyroidism type Ic defines a new subgroup of pseudohypoparathyroidism affecting selectively Gs $\alpha$-receptor interaction}

Thiele S, de Sanctis L, Werner R, Grotzinger J, Aydin C, Juppner H, Bastepe M, Hiort O

Department of Pediatrics and Adolescent Medicine, University of Lübeck, Germany.

thiele@paedia.ukl.mu-luebeck.de

Hum Mutat 2011;32:1-8.

Background: Pseudohypoparathyroidism type Ia (PHPIa) is caused by GNAS mutations leading to deficiency of the $\alpha$-subunit of stimulatory $\mathrm{G}$ proteins $(\mathrm{Gs} \alpha)$ that mediate signal transduction of G-proteincoupled receptors via cAMP. Both PHP type Ic (PHPIc) and PHPIa are clinically characterized by Albright hereditary osteodystrophy $(\mathrm{AHO})$ and with laboratory abnormalities consistent with peptide hormone resistance; however, in vitro activity of solubilized Gs $\alpha$ protein is reduced only in PHPIa but normal in PHPIc. 
Methods: The authors screened 32 patients with PHPIc for GNAS mutations. The identified three novel mutations and one PHPIa associated mutation were introduced into a wild-type Gs $\alpha$ expression vector and expressed in a Gs $\alpha$-null cell line. Receptor-mediated cAMP accumulation was investigated after stimulation of the endogenous $\beta_{2}$-adrenergic receptor, or the coexpressed PTH or TSH receptors; the synthesized cAMP was measured by RIA.

Results: Three GNAS mutations in four unrelated families with PHPIc were identified. Each of these mutants significantly reduced or completely disrupted receptor-mediated activation, but displayed normal receptor-independent activation, measured as cholera-toxin-induced cAMP accumulation. In contrast, the PHPIa-associated GNAS mutation disrupted both receptor-mediated activation and receptor-independent activation.

Conclusions: The authors conclude that their patients with PHPIc represent a new subgroup of PHP caused by Gs $\alpha$ deficiency due to selective defect in receptor-coupling functions of Gs $\alpha$.

Pseudohypoparathryoidism (PHP) describes a group of related disorders with end-organ resistance to PTH and other peptide hormones that mediate their actions through G-protein-coupled receptors via CAMP/protein kinase A. Based on clinical and laboratory features and of the result of Gs $\alpha$ protein activity in vitro measurement, PHP has been divided into different subgroups. The genetic background of the various forms differs. PHPla and pseudoPHP are caused by mutations in the GNAS gene exons 1-13; the phenotypic differences are due to imprinting and depend on whether the mutation resides on the maternal (PHPla) or paternal (pseudoPHP) allele. PHPIb, with hormone resistance but no AHO features, is caused by loss of methylation upstream of or within the GNAS locus. PHPIc shares similar clinical and biochemical features (AHO and hormone resistance) with PHPla but while the in vitro Gs $\alpha$ protein activity is diminished in PHPla, it is normal in PHPIc. It has been suggested that PHPIc may not be caused by functional impairment of the Gs $\alpha$ protein but by another component of the CAMP-dependent signaling pathway. In this paper, Thiele et al. show that a subgroup of patients with PHPIc do in fact have GNAS gene mutations despite normal Gs $\alpha$ activity and thus in some cases PHPIc is due to impaired Gs $\alpha$ function. The identified three mutations in 6 subjects (in a cohort of 32 patients with PHPIc) were shown to selectively affect receptor coupling but not adenylyl cyclaseactivating functions of Gs $\alpha$, while the PHPla-associated mutations affected both. These findings further elucidate the complexity of the genetic and molecular pathology underlying the PHP spectrum of diseases. The authors rightly conclude that a new classification of GNAS-related disorders should be proposed in the future, based not only on clinical and laboratory alterations, but also on molecular genetic, epigenetic and functional changes. Future studies are likely to expand our understanding on the pathogenesis of PHP as no genetic cause could be identified for most of the PHPIc subjects.

\section{Important for clinical practice \\ Safe and sure treatment for congenital rickets}

\section{Short- and long-term outcome of patients with pseudo-vitamin D deficiency rickets treated with calcitriol}

Edouard T, Alos N, Chabot G, Roughley P, Glorieux FH, Rauch F

Genetics Unit, Shriners Hospital for Children, Montreal, Que., Canada.

J Clin Endocrinol Metab 2011;96:82-9.

Background: Pseudo-vitamin D deficiency rickets (PDDR) is a rare autosomal recessive disorder with impaired synthesis of 1,25-dihydroxyvitamin $\mathrm{D}_{3}$ due to CYP27B1 gene mutations. Long-term effects of calcitriol treatment have not been reported.

Methods: The authors performed a retrospective review of clinical, biochemical and radiological data of 39 PDDR patients (20 females) who received calcitriol for 2.0-26 years, including 25 patients who had reached final height.

Results: Patients usually presented with active rickets, neurological signs, and short stature. Treatment with calcitriol resulted in normalization of biochemical parameters and lumbar spine bone mineral density within 3 months; height z-scores increased more slowly. Adult patients who commenced calcit- 
riol before pubertal growth spurt $(\mathrm{n}=11)$ had normal height, whereas patients treated first with highdose vitamin $\mathrm{D}$ and with calcitriol only after puberty $(\mathrm{n}=14)$ were shorter (height $\mathrm{z}$-score -2.2$)$. Lumbar spine bone mineral density was normal in all patients who had achieved final height. Nine women had 19 pregnancies; all were without complications and all newborns were eucalcemic at birth.

Conclusions: The authors conclude that treatment with calcitriol started in infancy results in short- and long-term correction of all clinical, biochemical, and radiological abnormalities related to PDDR, without serious adverse events.

This entity is known as vitamin D-dependent rickets, type $1 \mathrm{~A}$ and due to $1 \alpha$-hydroxylase deficiency. It is a rare form of inherited rickets caused by mutations in the gene encoding $1 \alpha$-hydroxylase (CYP27B1). Mutations lead to impaired synthesis of $1,25(\mathrm{OH})_{2} \mathrm{D}_{3}$ and to clinical and biochemical signs of rickets. To date, close to 100 patients have been reported but very little is known about the long-term outcome. The authors have carried out a careful chart review for clinical, biochemical and radiological parameters of 39 patients, followed in two centers in Montreal, Canada, and provide detailed analysis of the presenting symptoms, early response to treatment, evolution of growth and bone mineral density during childhood, and long-term outcome in adults. The take-home message is comforting: calcitriol therapy, started in early childhood, rapidly normalizes biochemistry, growth and bone mineralization without significant adverse effects (mild corneal calcium deposits in 1, mild nephrocalcinosis in 4 patients). Early diagnosis and therapy is essential to assure normal growth. The patients do not have increased bone fragility but may have dental enamel hypoplasia. Female mice with $1 \alpha$-hydroxylase deficiency develop, when untreated, uterine hypoplasia and absent corpora lutea and are infertile [14]. In contrast, female pubertal development, fertility and outcome of pregnancies was normal in the treated patients with $1 \alpha$-hydroxylase deficiency.

\section{Clinical trials, new treatments \\ Replacement therapy for hypoparathyroidism - injections or pills?}

\section{Long-term treatment of 12 children with chronic hypoparathyroidism: a randomized trial comparing synthetic human parathyroid hormone 1-34 versus calcitriol and calcium}

Winer KK, Sinaii N, Reynolds J, Peterson D, Dowdy K, Cutler GB, Jr.

The Eunice Kennedy Shriver National Institute of Child Health and Human Development, National Institutes of Health, Bethesda, Md., USA.

winer@mail.nih.gov

J Clin Endocrinol Metab 2010;95:2680-8.

Background: Hypoparathyroidism is among the few hormonal insufficiencies not treated with replacement of the missing hormone. The authors have carried out the first randomized controlled study comparing efficacy and safety of long-term synthetic human PTH 1-34 and calcitriol in children with hypoparathyroidism.

Methods: 12 children, aged 5-14 years, with chronic hypoparathyroidism were included in a 3-year randomized parallel trial comparing twice-daily calcitriol (plus calcium and cholecalciferol in four daily doses) vs. s.c. PTH 1-34 treatment.

Results: Mean predose serum calcium levels were maintained at, or just below, the normal range, and urine calcium levels remained in the normal range throughout the study; no significant differences were observed between the treatment groups. Creatinine clearance remained normal throughout the study. Bone turnover markers were mildly elevated during PTH 1-34 therapy and within the normal range during calcitriol therapy. Mean bone mineral density Z-scores and height and weight percentiles remained normal and did not differ between the groups throughout the 3-year follow-up.

Conclusion: The authors conclude that PTH 1-34 therapy is safe and effective in maintaining stable calcium homeostasis in children with hypoparathyroidism. The treatment allowed normal skeletal development and no differences in bone mineral accrual, linear growth, or weight gain between the two treatment arms were observed. 
It is remarkable how long it takes for an FDA-approved drug (since 2002) to come as a pilot very small scale study in children. Treatment of hypoparathryoidism may be challenging in children as undertreatment results in hypocalcemia and risk of seizures while overtreatment associates with nephrocalcinosis. Presently the treatment consists of active vitamin $\mathrm{D}$ analogs and calcium with phosphate-deficient diet. Yet, therapy mostly fails to lower serum phosphate levels. Moreover, PTH has important effects that are beyond its role in mineral metabolism. The authors of the present paper first carried out a 2-month pilot study in 2 adolescents with autoimmune hypoparathyroidism [15] and after these encouraging results and findings from adult studies [16] have now performed a 3-year randomized parallel trial comparing calcitriol (twice daily combined with calcium and cholecalciferol four times daily) and PTH 1-34 treatment. The authors rightly acknowledge that the study suffers from limited number of study subjects and relatively short duration of treatment in a lifelong disorder. Furthermore, the study protocol was very strict and it remains unknown whether the efficacy findings in a closely monitored research study in highly motivated families translates into realworld effectiveness in less closely monitored or less motivated families. Despite these limitations the results suggest that in selected cases, especially when conventional per oral treatment does not sufficiently prevent hypocalcemic episodes or is complicated by malabsorption (as is often case in APECED), subcutaneous PTH 1-34 treatment provides a safe and effective alternative.

\section{New treatments \\ Calcitonin against Phosphatonin - who wins?}

\section{Calcitonin administration in X-linked hypophosphatemia}

Liu ES, Carpenter TO, Gundberg CM, Simpson CA, Insogna KL

Yale University School of Medicine, New Haven, Conn., USA.

eva.s.liu@gmail.com

N Engl J Med 2011;364:1678-80.

Background: In X-linked hypophosphatemia (XLH), phosphate wasting results from increased levels of a circulating 'phosphatonin'. This phosphaturic factor is fibroblast growth factor 23 (FGF23), a bonederived protein with phosphaturic function.

Methods: In this brief Letter to the Editor, the authors report on the use of calcitonin in 7 untreated patients with XLH and in 6 controls.

Results: A single subcutaneous injection of 200 IU of calcitonin resulted in a significant drop in serum FGF23 at $4 \mathrm{~h}$ and the concentration remained below baseline for $16 \mathrm{~h}$ in XLH subjects. The 6 healthy controls showed no significant change in FGF23 levels after calcitonin injection. In patients with XLH the drop in serum FGF23 was associated with a significant increase in serum phosphate concentration, reaching a maximum of $129 \pm 16 \%$ of the baseline value at $10 \mathrm{~h}$; in the controls no change in serum phosphate was seen. Serum 1,25-OH2D levels rose similarly and significantly in both groups, and serum calcium and PTH increased only slightly in both groups. No significant change in either group was seen in renal tubular phosphate reabsorption.

Conclusions: In patients with XLH the researchers observed, after a single injection of calcitonin, a significant and sustained drop in circulating FGF23 and an increase in serum levels of phosphorus.

This interesting pilot study was prompted by two recent observations. Van Boekel et al. showed that administration of calcitonin to a patient with oncogenic osteomalacia caused a drop in serum FGF23 and Gooi et al. reported that osteocytes express the calcitonin receptor and respond to calcitonin [17, 18]. The researchers concluded that since the osteocytes are the primary source of FGF23, the effects of calcitonin seen in their patients with XLH - the decreased FGF23 and increased serum phosphorus - directly result from normalization of osteocyte FGF23 production. In XLH 1,25- $\mathrm{OH}_{2} \mathrm{D}$ is inappropriately normal despite hypophosphatemia. Calcitonin stimulated renal $1 \alpha$-hydroxylase and resulted in an increase in serum $1,25-\mathrm{OH}_{2} \mathrm{D}$ in the XLH patients and the controls. The authors speculate that the findings raise the possibility that calcitonin may be a therapeutic option for patients with XLH. New therapies would indeed be greatly needed as the conventional therapy with oral phosphate in 
multiple daily doses combined with active vitamin D often results in unsatisfactory clinical response, associates with complications and is demanding for the patient and the parents. Innovative studies like this, with 'old medications', and preliminary observations on the use of FGF23 antibody in animal models [19] pave the way for improved therapy for phosphate-wasting disorders.

\title{
Concepts revised \\ Osteoclasts need their daily vitamin D
}

\section{Osteoclastic metabolism of $25(\mathrm{OH})$-vitamin $\mathrm{D}_{3}$ : a potential mechanism for optimization of bone resorption}

\author{
Kogawa M, Findlay DM, Anderson PH, Ormsby R, Vincent C, Morris HA, Atkins GJ \\ Bone Cell Biology Group, Discipline of Orthopaedics and Trauma, University of Adelaide, Adelaide, S.A., Australia. \\ Endocrinology 2010;151:4613-25.
}

Background: Extrarenal synthesis of 1,25-dihydroxyvitamin $\mathrm{D}_{3}(1,25-\mathrm{D})$ has been demonstrated in several cell types including osteoblasts. Human and rodent studies suggest that 25 -hydroxyvitamin $\mathrm{D}_{3}(25-\mathrm{D})$, rather than 1,25-D, directly affects bone mineralization. It is not known how osteoclasts participate in local production of $1,25-\mathrm{D}$.

Methods: The authors examined vitamin D metabolism during osteoclastogenesis in human peripheral blood mononuclear cells, mouse osteoclast precursor cell line and in giant cell tumor cells.

Results: CYP27B1 (1 $\alpha$-hydroxylase) mRNA expression increased with exposure of PBMCs to macrophage colony-stimulating factor. Consistent with this, human osteoclast cultures incubated with 25-D produced measurable quantities of 1,25-D. Osteoclast formation in the presence of physiological concentrations of 25-D resulted in significant upregulation of an osteoclast transcription factor and key osteoclast marker genes in the studied cell lines. The expression of the osteoblast-coupling factor, ephrin-b2, was also increased in the presence of 25-D. Conversely, selective silencing of CYP27B1 in osteoclast precursor cells decreased their osteoclastogenic potential. 25-D dose-dependently reduced the resorptive capacity of osteoclasts without compromising cell viability.

Conclusions: The authors conclude that 25-D metabolism is an important intrinsic mechanism for optimizing osteoclast differentiation, ameliorating osteoclast activity, and potentially promoting the coupling of bone resorption to formation.

There is growing evidence that the skeleton is an intracrine organ for vitamin D metabolism. Recent studies suggest that circulating $25-D$, rather than $1,25-D$, directly affects bone mineralization. The present study indicates that not only osteoblasts but also osteoclasts participate in local production of 1,25-D. The CYP27B1 mRNA expression increases with the differentiation of macrophages and osteoclasts. The metabolism of 25-D into 1,25-D by human osteoclast precursors resulted in increased expression of a number of important osteoclast-associated genes. Although formation markers increased, osteoclast activity was inhibited in the presence of physiologically replete levels of 25-D: increasing 25-D concentrations dose-dependently inhibited resorption with a maximal inhibitory effect at $50 \mathrm{~nm}$, corresponding to the physiological threshold level of 25-D, which is presently considered sufficient for optimal skeletal health [20]. The findings further suggest that 25-D might serve to optimize local cell-to-cell communication between osteoclasts and osteoblasts. Based on these and previous findings, adequate levels of 25-D, and skeletal metabolism of 25-D, may be important for optimal development and function of several skeletal aspects. Although the gross effects of vitamin $\mathrm{D}$ deficiency on bone in vivo can be corrected by administering calcium and phosphate, there is increasing evidence to indicate that during normal bone metabolism, vitamin $D$ plays a direct role in optimizing the activity of bone cells, as recently shown [21]. 


\section{Circulating levels of soluble klotho and FGF23 in X-linked hypophosphatemia: circadian variance, effects of treatment, and relationship to parathyroid status}

Carpenter TO, Insogna KL, Zhang JH, Ellis B, Nieman S, Simpson C, Olear E, Gundberg CM

Department of Pediatrics, Yale University School of Medicine, New Haven, Conn., USA.

thomas.carpenter@yale.edu

J Clin Endocrinol Metab 2010;95:E352-7.

Background: Elevated circulating fibroblast growth factor 23 (FGF23) levels have been reported in $\mathrm{X}$-linked hypophosphatemia (XLH). Klotho is critical for FGF23 signaling and renal phosphate handling by forming a ternary ligand-binding complex with FGF receptors. The purpose of this study was to characterize circulating FGF23 and klotho in XLH.

Methods: 23 individuals, children and adults, with XLH and 8 controls were included. Subjects with XLH withheld medication for 14 days. Fasting serum FGF23, PTH, klotho, phosphate, and 1,25-dihydroxyvitamin D were obtained. Adults were also sampled during XLH treatment, and circadian sampling was performed in selected individuals.

Results: FGF23 levels were higher in XLH than in controls, and in treated XLH subjects compared to XLH subjects not receiving medical therapy. Children had higher klotho levels than adults, but values in XLH were similar to controls. A strong positive correlation between FGF23 and PTH was found in XLH subjects but not in controls. Circulating klotho, but not FGF23, had a diurnal pattern, with an approximately $40 \%$ reduction in klotho concentration at midnight, coinciding with a peak in serum phosphate.

Conclusions: Serum klotho declines with age and demonstrates circadian variation but is normal in XLH. Serum FGF23 is elevated in XLH and further increases with therapy, but demonstrates no diurnal variation and is similar in children and adults. FGF23 and PTH showed direct correlation in subjects with XLH suggesting that FGF23-associated PTH regulation is aberrant in this disorder.

Several new regulators of phosphate metabolism have recently been discovered. Elevated circulating FGF23 levels have been reported in X-linked, autosomal dominant and autosomal recessive forms of hypophosphatemia as well as in other hypophosphatemic states. Klotho, a membrane protein, is critical for FGF23 signaling in the renal proximal tubule as klotho and FGF receptor 1 form a heterodimeric, high-affinity receptor for FGF23. Klotho-null mice have elevated serum phosphate and 1,25-dihydroxyvitamin $D$ levels. Klotho is expressed in renal tubules but also in the pituitary (function unknown) and parathyroid gland, where FGF23 appears to reduce PTH secretion. Apart from functions in signaling, klotho may also function as a hormone; the extracellular domain of klotho protein is shed from the cell surface and this soluble form acts as a humoral factor that regulates multiple growth factor signaling pathways. In this study, Carpenter et al. show age-related changes in serum klotho concentration, with children having significantly greater levels than adults do, as well as a significant circadian variation. Klotho behaved no differently in XLH subjects and healthy controls. Future studies are likely to find more target functions for klotho.

References

1. Lee NK, Sowa H, Hinoi E, Ferron M, Ahn JD, Confavreux C, et al: Endocrine regulation of energy metabolism by the skeleton. Cell 2007;130:456-469.

2. Chrysis D, Heino T, Sävendahl L: Growth plate, bone and calcium; in Carel J-C, Hochberg Z (eds): Yearbook of Pediatric Endocrinology 2008. Basel, Karger, 2008, pp 63-79.

3. Forsberg JA, Pepek JM, Wagner S, Wilson K, Flint J, Andersen RC, et al: Heterotopic ossification in high-energy wartime extremity injuries: prevalence and risk factors. J Bone Joint Surg Am 2009;91:1084-1091.

4. Black DM, Delmas PD, Eastell R, Reid IR, Boonen S, Cauley JA, et al: Once-yearly zoledronic acid for treatment of postmenopausal osteoporosis. N Engl J Med 2007;356:1809-1822.

5. Vahle JL, Sato M, Long GG, Young JK, Francis PC, Engelhardt JA, et al: Skeletal changes in rats given daily subcutaneous injections of recombinant human parathyroid hormone (1-34) for 2 years and relevance to human safety. Toxicol Pathol 2002;30:312-321.

6. Tao J, Chen S, Lee B: Alteration of Notch signaling in skeletal development and disease. Ann N Y Acad Sci 2010;1192:257268. 
7. Isidor B, Lindenbaum P, Pichon O, Bezieau S, Dina C, Jacquemont S, et al: Truncating mutations in the last exon of NOTCH2 cause a rare skeletal disorder with osteoporosis. Nat Genet 2011;43:306-308.

8. Richards JB, Kavvoura FK, Rivadeneira F, Styrkarsdottir U, Estrada K, Halldorsson BV, et al: Collaborative meta-analysis: associations of 150 candidate genes with osteoporosis and osteoporotic fracture. Ann Intern Med 2009;151:528537.

9. Styrkarsdottir U, Halldorsson BV, Gretarsdottir S, Gudbjartsson DF, Walters GB, Ingvarsson T, et al: Multiple genetic loci for bone mineral density and fractures. N Engl J Med 2008;358:2355-2365.

10. Chrysis D, Heino T, Sävendahl L. Growth plate, bone and calcium; in Carel J-C, Hochberg Z (eds): Yearbook of Pediatric Endocrinology 2007. Basel, Karger, 2007.

11. Heino T, Tiosano D, Qawlik A, Sävendahl L: Bone, growth plate and mineral metabolism; in Carel J-C, Hochberg Z (eds): Yearbook of Pediatric Endocrinology 2010. Basel, Karger, 2010, pp 65-81.

12. Kronenberg HM: Developmental regulation of the growth plate. Nature 2003;423:332-336.

13. Sakamoto A, Chen M, Kobayashi T, Kronenberg HM, Weinstein LS: Chondrocyte-specific knockout of the G-protein $\mathrm{G}(\mathrm{s}) \alpha$ leads to epiphyseal and growth plate abnormalities and ectopic chondrocyte formation. J Bone Miner Res 2005;20:663-671.

14. Panda DK, Miao D, Tremblay ML, Sirois J, Farookhi R, Hendy GN, et al: Targeted ablation of the 25-hydroxyvitamin D $1 \alpha$-hydroxylase enzyme: evidence for skeletal, reproductive, and immune dysfunction. Proc Natl Acad Sci U S A 2001;98:7498-7503.

15. Stogmann W, Bohrn E, Woloszczuk W: Initial experiences with substitution treatment of hypoparathyroidism with synthetic human parathyroid hormone. Monatsschr Kinderheilkd 1990;138:141-146.

16. Winer KK, Ko CW, Reynolds JC, Dowdy K, Keil M, Peterson D, et al: Long-term treatment of hypoparathyroidism: a randomized controlled study comparing parathyroid hormone-(1-34) versus calcitriol and calcium. J Clin Endocrinol Metab 2003;88:4214-4220.

17. Van Boekel G, Ruinemans-Koerts J, Joosten F, Dijkhuizen P, van Sorge A, de Boer H: Tumor producing fibroblast growth factor 23 localized by two-staged venous sampling. Eur J Endocrinol 2008;158:431-437.

18. Gooi JH, Pompolo S, Karsdal MA, Kulkarni NH, Kalajzic I, McAhren SH, et al: Calcitonin impairs the anabolic effect of PTH in young rats and stimulates expression of sclerostin by osteocytes. Bone 2010;46:1486-1497.

19. Aono Y, Yamazaki Y, Yasutake J, Kawata T, Hasegawa H, Urakawa I, et al: Therapeutic effects of anti-FGF23 antibodies in hypophosphatemic rickets/osteomalacia. J Bone Miner Res 2009;24:1879-1888.

20. Misra M, Pacaud D, Petryk A, Collett-Solberg PF, Kappy M: Vitamin D deficiency in children and its management: review of current knowledge and recommendations. Pediatrics 2008;122:398-417.

21. Anderson PH, Atkins GJ, Turner AG, Kogawa M, Findlay DM, Morris HA: Vitamin D metabolism within bone cells: Effects on bone structure and strength. Mol Cell Endocrinol 2011 (E-pub ahead of print). 